\title{
Combined lumped element network and transmission line synthesis for passive microwave structures
}

\author{
J. A. Russer ${ }^{1}$, F. Mukhtar ${ }^{1}$, A. Baev ${ }^{2}$, Y. Kuznetsov ${ }^{2}$, and P. Russer ${ }^{1}$ \\ ${ }^{1}$ Lehrstuhl für Nanoelektronik, Technische Universität München, Arcisstrasse 21, Munich, Germany \\ ${ }^{2}$ Theoretical Radio Engineering Dept., Moscow Aviation Inst., Volokolamskoe shosse, 4, GSP-3, Moscow, 125993, Russia
}

\begin{abstract}
Compact circuit models of electromagnetic structures are a valuable tool for embedding distributed circuits into complex circuits and systems. However, electromagnetic structures with large internal propagation delay are described by impedance functions with a large number of frequency poles in a given frequency interval and therefore yielding equivalent circuit models with a high number of lumped circuit elements. The number of circuit elements can be reduced considerably if in addition to capacitors, inductors, resistors and ideal transformers also delay lines are included. In this contribution a systematic procedure for the generation of combined lumped element/delay line equivalent circuit models on the basis of numerical data is described. The numerical data are obtained by numerical fullwave modeling of the electromagnetic structure. The simulation results are decomposed into two parts representing a lumped elements model and a delay line model. The extraction of the model parameters is performed by application of the system identification procedure to the scattering transfer function. Examples for the modeling of electromagnetic structures are presented.
\end{abstract}

\section{Introduction}

The importance for applying circuit-theoretic multi-port concepts in the modeling of wireless communication links already has been pointed out in Ivrlac and Nossek (2010). Different from simple information theoretic models multi-port models allow to consider the energy flow and the coupling of antenna elements. The use two-port models of antennas already has been discussed in literature (Rogers et al., 2003; Boryssenko and Schaubert, 2007; Aberle, 2008). These models yielded an improved description of antennas, however,

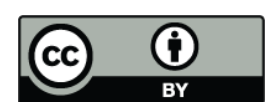

Correspondence to: J. A. Russer (jrusser@tum.de) due to the limited number of lumped elements the antenna could be modeled in a narrow frequency band only.

Distributed circuits can be modeled also in a broad frequency band with arbitrary accuracy using lumped element network models. A general way to establish network models is based on modal analysis and similar techniques (Russer, 2006; Felsen et al., 2009; Russer et al., 2010). In Russer et al. (2010) also the modeling of lossy structures on the basis of Brune's equivalent circuit realization has been discussed. However, electromagnetic structures with large internal propagation delay are described by impedance functions with a large number of frequency poles in a given frequency interval and therefore yielding equivalent circuit models with a high number of lumped circuit elements. The number of circuit elements can be reduced considerably if in addition to capacitors, inductors, resistors and ideal transformers also delay lines are included. A combined lumped elements and delay line approach already has been presented in Shevgunov et al. (2008).

In this contribution a systematic procedure for the generation of a combined lumped element/delay line equivalent circuit model for a wireless transmission link on the basis of numerical data is described. The numerical data are obtained by numerical full-wave modeling of the electromagnetic structure. The simulation results are decomposed into two parts representing a lumped elements model and a delay line model. The extraction of the model parameters is performed by application of the system identification procedure to the scattering transfer function. Examples for the modeling of electromagnetic structures are presented.

\section{Model of a wireless link}

Let us consider the easiest case of a wireless transmission link consisting of two antennas connected in the far-field over free-space. Figure 1 shows this arrangement schematically. In $\boldsymbol{Z}$ representation the voltage-current relations are given by

Published by Copernicus Publications on behalf of the URSI Landesausschuss in der Bundesrepublik Deutschland e.V. 

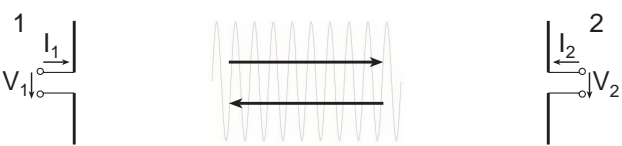

Fig. 1. Wireless transmission link with two antennas.

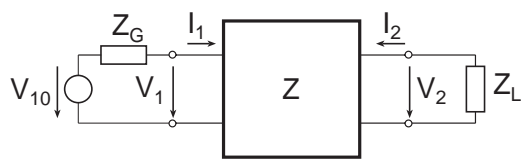

Fig. 2. Two-port representation of the wireless transmission link with source and load.

$$
\left[\begin{array}{l}
V_{1} \\
V_{2}
\end{array}\right]=\left[\begin{array}{ll}
Z_{11} & Z_{12} \\
Z_{21} & Z_{22}
\end{array}\right]\left[\begin{array}{l}
I_{1} \\
I_{2}
\end{array}\right]
$$

Figure 2 shows the block diagram of the wireless link for the case where antenna 1 is the transmitting antenna and antenna 2 is the receiving antenna. The transmitter has the open source voltage $V_{10}$ and the source impedance $Z_{\mathrm{G}}$. The receiving antenna is terminated with the load impedance $Z_{\mathrm{L}}$. Under these conditions the input and output currents $I_{1}$ and $I_{2}$, respectively are given by

$$
\begin{aligned}
& I_{1}=\frac{Z_{22}+Z_{\mathrm{L}}}{\left(Z_{11}+Z_{\mathrm{G}}\right)\left(Z_{22}+Z_{\mathrm{L}}\right)-Z_{12} Z_{21}} V_{10}, \\
& I_{2}=\frac{Z_{21}}{\left(Z_{11}+Z_{\mathrm{G}}\right)\left(Z_{22}+Z_{\mathrm{L}}\right)-Z_{12} Z_{21}} V_{10} .
\end{aligned}
$$

Figure 3 shows the schematic two-port representation of the link with source and load where $Z_{12}$ and $Z_{21}$ are represented by current controlled voltage sources. The input impedance $Z_{\text {in }}$ of the wireless link two-port $\boldsymbol{Z}$ terminated by $Z_{\mathrm{L}}$ is given by

$Z_{\text {in }}=Z_{11}-\frac{Z_{12} Z_{21}}{Z_{22}+Z_{\mathrm{L}}}$.

The available power $P_{\mathrm{a}}$ of the transmitter is given by

$P_{\mathrm{a}}=\frac{\left|V_{10}\right|^{2}}{8 \Re\left\{Z_{\mathrm{G}}\right\}}$.

The power delivered to the load $R_{\mathrm{L}}$ is

$P_{\mathrm{L}}=\frac{1}{2} \Re\left\{Z_{\mathrm{L}}\right\}\left|I_{2}\right|^{2}$.

The transducer power gain is defined as

$G_{\mathrm{t}}=\frac{P_{\mathrm{L}}}{P_{\mathrm{a}}}=\frac{4\left|Z_{21}\right|^{2} \Re\left\{Z_{\mathrm{G}}\right\} \Re\left\{Z_{\mathrm{L}}\right\}}{\left|\left(Z_{11}+Z_{\mathrm{G}}\right)\left(Z_{22}+Z_{\mathrm{L}}\right)-Z_{12} Z_{21}\right|^{2}}$.

In a wireless transmission link the reaction of the receiving antenna on the transmitting antenna usually is negligible, i.e. $\left|\left(Z_{11}+Z_{\mathrm{G}}\right) Z_{22}\right| \gg\left|Z_{12} Z_{21}\right|$. In this case we can approximate the transducer power gain by the unilateral power gain.

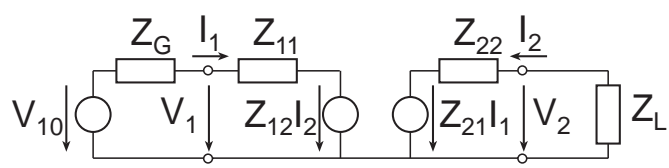

Fig. 3. Two-port representation of the wireless transmission link with source and load where $Z_{12}$ and $Z_{21}$ are represented by current controlled voltage sources.
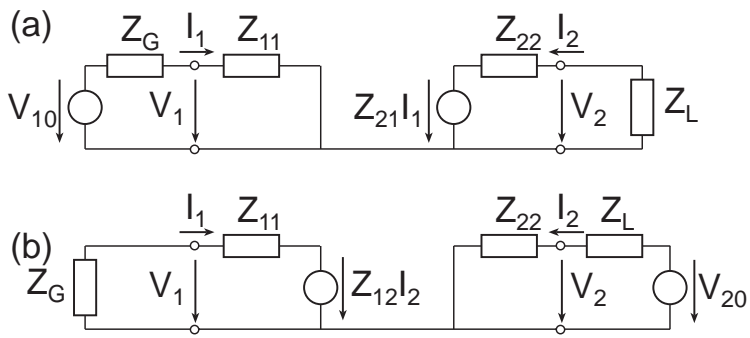

Fig. 4. Two-port representation of the unilateralized wireless transmission link with source and load (a) with antenna 1 as the transmitting antenna, (b) with antenna 2 as the transmitting antenna.

Depending on the direction of transmission we can replace the reciprocal wireless link models by the non-reciprocal models shown in Fig. 4. In this case the power gain $G_{\mathrm{t}}$ is approximated by the unilateral power gain

$G_{\mathrm{tu}}=\frac{4\left|Z_{21}\right|^{2} \Re\left\{Z_{\mathrm{G}}\right\} \Re\left\{Z_{\mathrm{L}}\right\}}{\left|\left(Z_{11}+Z_{\mathrm{G}}\right)\left(Z_{22}+Z_{\mathrm{L}}\right)\right|^{2}}$.

We note that in this approximation the antenna feed impedances of antenna 1 and antenna 2 are given by $Z_{11}$ and $Z_{22}$ respectively.

The unilateral power gain exhibits its maximum value $G_{\text {tu,max }}$ for power matching at the input and output, i.e. $Z_{\mathrm{G}}=Z_{11}^{*}, Z_{\mathrm{L}}=Z_{22}^{*}$ and is given by

$G_{\mathrm{tu}, \max }=\frac{\left|Z_{21}\right|^{2}}{4 \Re\left\{Z_{11}\right\} \mathfrak{R}\left\{Z_{22}\right\}}$.

\section{The two-port antenna model}

As long as the coupling of the antenna with the other antenna is neglected the antenna can be considered as a passive oneport. If the antenna is coupled with one or more other antennas over the far-field only, the driving point impedance $Z_{\text {in }}$ of this antenna one-port will not be influenced by the other antennas. In the following we assume the antenna itself to be lossless. The real part of the antenna driving point impedance in that case is only due to the active power radiated by the antenna. $Z_{\text {in }}$ is a positive real impedance function. A oneport with a positive real impedance function can be represented by a reactive reciprocal two-port $\boldsymbol{Z}_{\mathbf{R}}$ terminated by a real resistor $R_{\mathrm{r}}$ (Guillemin, 1957). Figure 5 shows the block 


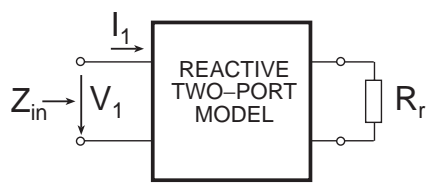

Fig. 5. Two-port antenna model.

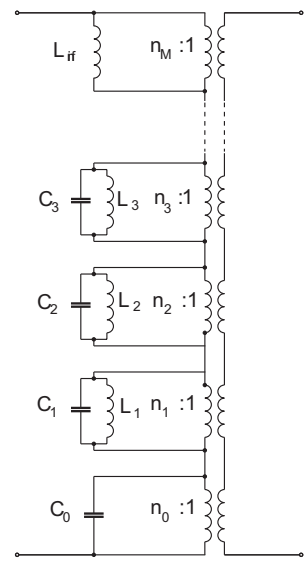

Fig. 6. Foster realization of the reactive two-port.

diagram of the two-port antenna model. The determination of the positive rational impedance functions of the two-port was performed by system identification methods (Kuznetsov et al., 2004; Russer et al., 2010).

An approximation of the lossless reciprocal two-port based on a finite number of poles of its two-port reactance matrix $\boldsymbol{Z}_{\mathbf{R}}$ can be realized with a finite number of capacitors, inductors and ideal transformers. Figure 6 shows the canonical Foster realization of the reactive two-port (Russer, 2006; Felsen et al., 2009; Russer et al., 2010).

The complete impedance matrix describing a circuit with a finite number of poles is obtained by parallel connecting the circuits describing the individual poles. In the canonical Foster representation, the impedance matrix $\boldsymbol{Z}(\omega)$ is given by

$\boldsymbol{Z}_{\lambda}(\omega)=\frac{1}{j \omega C_{0}} \boldsymbol{B}_{0}+\sum_{\lambda=1}^{N} \frac{1}{j \omega C_{\lambda}} \frac{\omega^{2}}{\omega^{2}-\omega_{\lambda}^{2}} \boldsymbol{B}_{\lambda}+j \omega L_{\infty} \boldsymbol{B}_{\infty}$.

The frequency independent rank 1 matrices $\boldsymbol{B}_{\lambda}$ are given by

$\boldsymbol{B}_{\lambda}=\left[\begin{array}{cc}n_{\lambda}^{2} & n_{\lambda} \\ n_{\lambda} & 1\end{array}\right]$

where the $n_{\lambda}$ are the turns ratios of the transformers.

We have applied the methods described in this paper to model communication links between monolithic integrated on-chip antennas. Figure 7 shows a photograph of an openchip antenna with CMOS circuits under the antenna electrode (Yordanov and Russer, 2010). The antennas have been measured on-wafer and diced.

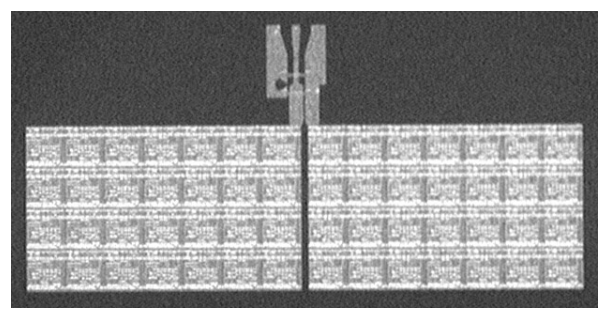

Fig. 7. Photograph of the open slot antenna (Yordanov and Russer, 2010).

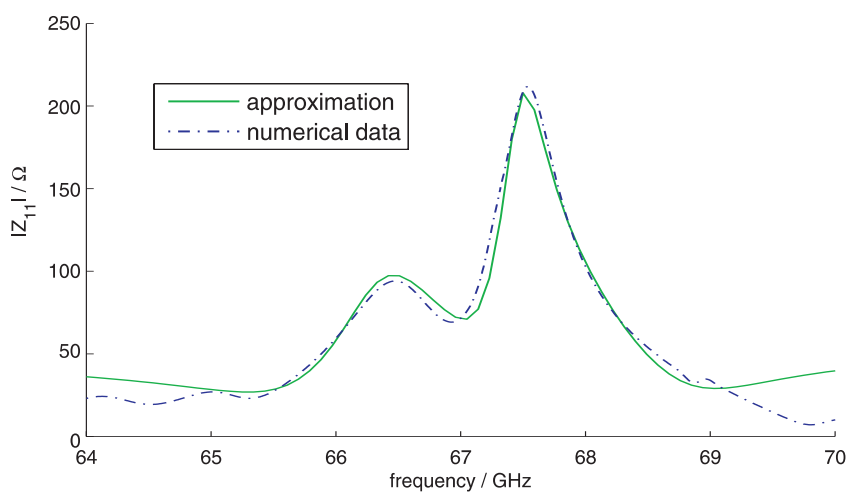

Fig. 8. $\left|Z_{11}\right|$ of the wireless transmission link.

Figure 8 shows the comparison of the $\left|Z_{11}\right|$ data obtained from numerical simulation with the data computed from the two-port model according to Fig. 5 with the reactive Foster two-port model shown in Fig. 6. The numerical full-wave simulations have been performed using CST software. Based on four pairs of poles and two single poles at zero and infinity the two-port model provides an accurate model of $Z_{11}$ for the frequency band from $65 \mathrm{GHz}$ to $69 \mathrm{GHz}$. The frequency range may be extended by increasing the number of poles.

\section{The wireless link model}

To extend the two-port antenna model to the full wireless link model we start from the assumption that the losses in the antenna are mainly radiation losses. In this case we can assume that the reactive two-port in Fig. 6 models the nearfield of the antenna whereas the real resistor $R_{\mathrm{r}}$ models the energy dissipation in the far-field. Considering dispersionfree single-path propagation between the two antennas, we can model the free-space propagation of the electromagnetic wave by a dispersion-free lossy transmission line (TL). The length of this transmission line corresponds to the distance between the antennas and the attenuation follows from Friis transmission formula (Russer, 2006).

This yields the model of the complete wireless transmission link shown in Fig. 9. The scattering matrix of the lossy 


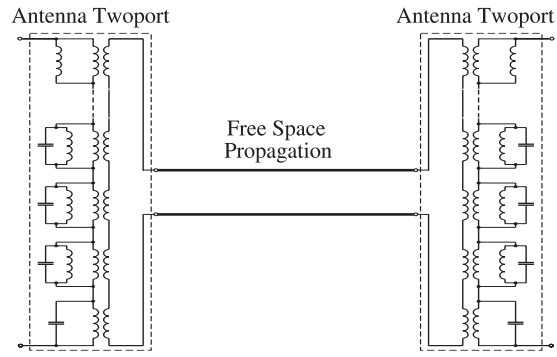

Fig. 9. Model of the complete wireless transmission link.

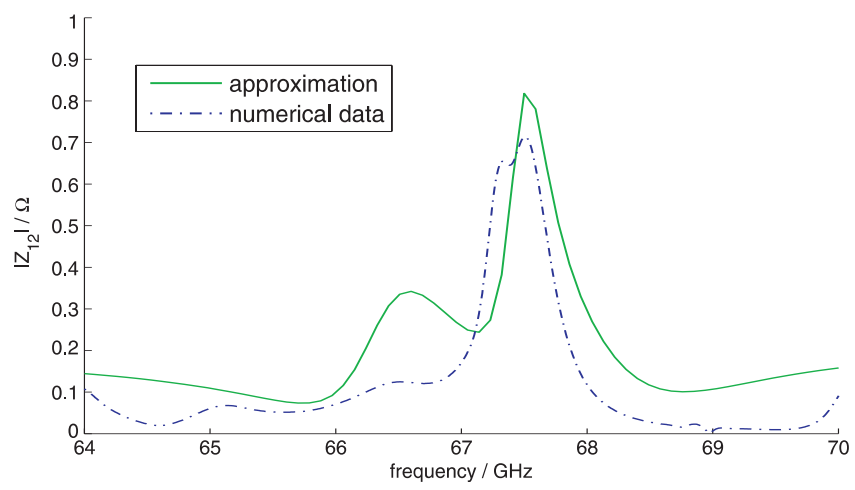

Fig. 10. $\left|Z_{12}\right|$ of the wireless transmission link.

transmission line with wave impedance $R_{\mathrm{r}}$ is given by

$S_{\mathrm{L}}=a \frac{\exp (-j k r)}{k r}\left[\begin{array}{ll}0 & 1 \\ 1 & 0\end{array}\right]$

where $a$ represents the attenuation according to the Friis formula and $r$ is the distance between the antennas. The corresponding $\boldsymbol{Z}$-matrix is given by

$Z_{\mathrm{L}}=Z_{0}\left[\begin{array}{cc}\frac{1+\frac{a^{2} \exp (-2 j k r)}{(k r)^{2}}}{1-\frac{a^{2} \exp (-2 j k r)}{(k r)^{2}}} & \frac{a \exp (-j k r)}{k r} \\ \frac{a \exp (--k r)}{k r} & 1-\frac{a^{2} \exp (-2 j k r)}{(k r r)} \\ \frac{a^{2} \exp (-2 j k r)}{(k r)^{2}} \\ 1-\frac{a^{2} \exp (-2 j k r)}{(k r)^{2}} & \frac{a^{2} \exp (-2 j k r)}{(k r)^{2}}\end{array}\right]$.

For $\frac{a \exp (-j k r)}{k r} \ll 1$ this can be approximated by

$Z_{\mathrm{L}} \approx Z_{0}\left[\begin{array}{cc}1 & \frac{a \exp (-j k r)}{k r} \\ \frac{a \exp (-j k r)}{k r} & 1\end{array}\right]$.

Figure 10 shows the comparison of the numerical data of $\left|Z_{12}\right|$ obtained from the full-wave simulation of the wireless transmission link with the data computed from the model shown in Fig. 9. The stronger deviation between these data, compared with the case of $\left|Z_{11}\right|$ may be due to losses in the antenna and will be subject to further investigations.

\section{Conclusions}

We have presented an accurate method for generation of compact circuit models of wireless transmission links, where the antenna structure and the near-field regions are modeled by reactive lumped element networks and the far-field propagation by dispersion-free lossy transmission lines. Further investigations will extend this work towards multi-antenna systems and multi-path propagation. This should provide a framework for physical modeling of MIMO systems.

Acknowledgements. This work was supported by the Deutsche Forschungsgemeinschaft.

\section{References}

Aberle, J.: Two-Port Representation of an Antenna With Application to Non-Foster Matching Networks, Antennas and Propagation, IEEE Transactions on, 56, 1218-1222, 2008.

Boryssenko, A. O. and Schaubert, D. H.: On optimal port loading conditions for maximizing product of energy gain and bandwidth in broadband antenna links, IEEE Transactions on Antennas and Propagation, 55, 3668-3676, 2007.

Felsen, L. B., Mongiardo, M., and Russer, P.: Electromagnetic Field Computation by Network Methods, Springer, Berlin, Germany, 2009.

Guillemin, E. A.: Synthesis of Passive Networks, Wiley, New York, 1957.

Ivrlac, M. T. and Nossek, J. A.: Toward a Circuit Theory of Communication, Circuits and Systems I: Regular Papers, IEEE Transactions on, 57, 1663-1683, 2010.

Kuznetsov, Y., Baev, A., Coccetti, F., and Russer, P.: The Ultra Wideband Transfer Function Representation of Complex ThreeDimensional Electromagnetic Structures, in: 2004 International Symposium on Signals, Systems, and Electronics ISSSE'04, August 10-13, Linz, Austria, 2004.

Rogers, S. D., Aberle, J. T., and Auckland, D. T.: Two-port model of an antenna for use in characterizing wireless communications systems, obtained using efficiency measurements, IEEE Antennas and Propagation Magazine, 45, 115-118, 2003.

Russer, P.: Electromagnetics, Microwave Circuit and Antenna Design for Communications Engineering, Artech House, Boston, second edn., 2006.

Russer, J. A., Kuznetsov, Y., and Russer, P.: Discrete-time network and state equation methods applied to computational electromagnetics, Mikrotalasna Revija (Microwave Review), pp. 2 14,2010

Shevgunov, T., Baev, A., Kuznetsov, Y., and Russer, P.: Lumped element network synthesis for one-port passive microwave structures, in: Microwaves, Radar and Wireless Communications, 2008. MIKON 2008. 17th International Conference on, pp. 14, 2008

Yordanov, H. and Russer, P.: Area-Efficient Integrated Antennas for Inter-Chip Communication, in: Proceedings of the 40th European Microwave Conference, EuMC 2010, Paris, France, 2010. 